\title{
Comparative study between chemiluminescence assay and two different sensitive polymerase chain reactions on the diagnosis of serial herpes simplex virus encephalitis
}

\author{
S Kamei, T Takasu, T Morishima, T Yoshihara, T Tetsuka
}

\begin{abstract}
Objective-A prospective study was undertaken on the diagnosis of herpes simplex encephalitis (HSVE) by comparing chemiluminescence assay (CL) and two different sensitive polymerase chain reactions (PCRs).
\end{abstract}

Methods-The materials comprised 53 serial CSF samples from 31 patients with acute encephalitis with suspected HSVE. Each CSF was distributed to three independent laboratories to perform quantitative measurements by $C L$, the low sensitive (single) PCR, and high sensitive (nested) PCR. The CL provided a method of detecting HSV itself and the small fragment with HSV antigenicity which was composed of viral component proteins. The serial CSFs were found retrospectively to comprise 24 samples from 11 patients with HSVE due to HSV1 and 29 samples from 20 patients with non-HSVE. Results- the CL showed 50 to 48000 pfu/ml in all samples of HSVE (except one) taken from the 3 rd to the 25 th day. The low sensitive PCR demonstrated 50 to $47000 \mathrm{pfu} / \mathrm{ml}$ in only six samples of HSVE. The high sensitive PCR disclosed less than 100 to 120000 copies $/ \mathrm{ml}$ in 11 samples of HSVE. At the acute stage from the 1 st to 7th day, the sensitivities of $\mathrm{CL}$ and the high sensitive PCR were $100 \%$, but that of the low sensitive PCR was $75 \%$. The sensitivity of CL was significantly higher than those of both PCRs after the acute stage on the 15 th to 32 nd day. The specificities and positive predictive values of the three methods were $100 \%$. However, the negative predictive value of CL was significantly higher than that of the low sensitive PCR.

Conclusions-The sensitivity of $\mathrm{CL}$ is equivalent to that of the high sensitive PCR during the acute stage and significantly higher than that of the high sensitive PCR after the acute stage. A clear difference in sensitivity exists between the different PCRs. A combination of the PCR, chemiluminescence assay, and serological antibody diagnosis is currently considered the most effective approach for the clinical diagnosis of HSVE.

(f Neurol Neurosurg Psychiatry 1999;67:596-601)

Keywords: chemiluminescence; PCR; herpes simplex virus encephalitis
Quantitative detection of intrathecal antigen employing the chemiluminescence assay provides a highly sensitive and specific tool for the early diagnosis of herpes simplex virus encephalitis (HSVE), and has the potential to detect the viral antigen quantitatively. ${ }^{1}$ Moreover, the assay requires only 5 hours to yield its results. Detection of the HSV genome sequence in the CSF by the polymerase chain reaction (PCR) has been established as a useful diagnostic method at the acute stage of HSVE. $^{2-5}$ The PCR represents a highly sensitive and specific diagnostic technique for HSVE by detecting the HSV genome sequence in the CSF. Recently, quantitative detection of the HSV selected genome sequence by the PCR has been reported..$^{6-8}$ The purpose of the present prospective study was to compare the potential for diagnosing HSVE between quantitative detection of the intrathecal antigen by chemiluminescence assay and quantitative detection of the intrathecal viral genome sequence by the single and nested PCRs.

Subjects and methods PATIENTS AND SPECIMENS

The materials for examination consisted of 53 CSF samples from 31 serial patients with acute encephalitis who were initially suspected of having HSVE, at four registered hospitals (Nihon University Itabashi Hospital and three other affiliated hospitals) during 1994 to 1998. After CSF sampling in each of the patients, the materials obtained were immediately distributed to three different laboratories to perform measurements by chemiluminescence assay (S Kamei, Department of Neurology, Nihon University School of Medicine, Tokyo), the low sensitive PCR (T Yoshihara, Center for Molecular Biology and Cytogenetics, SRL Inc, Tokyo), and the high sensitive PCR (T Morishima, Department of Pediatrics, Nagoya University School of Medicine, Nagoya), independently. The samples were transported to the three independent laboratories at $-20^{\circ} \mathrm{C}$ within 24 hours. There was thus no available information concerning serological data for each patient at the time of measurement at these laboratories: all CSF samples were blinded for their aetiological diagnosis before they were assayed. The diagnosis of an acute episode of encephalitis as HSVE was confirmed retrospectively based on the detection of specific intrathecal HSV antibody synthesis.

Such synthesis was evaluated from the antibody ratio (serum/CSF antibody $\leqslant 20$ ), ${ }^{9}$ 
antibody index (CSF/serum antibody/CSF/ serum albumin $>1.91)^{10}$ and capture enzyme linked immunosorbent assay (c-EIA) ratio $(\mathrm{CSF} / \mathrm{serum}$ antibody $\geqslant 1),{ }^{11}$ as described previously. All patients with HSVE had positive results for both the antibody ratio and c-EIA ratio. All patients with HSVE, except two patients (Nos 2 and 5), who had not been examined for their albumin concentration, showed positive results for the antibody index also. Rheumatoid factor and varicella zoster virus antibody were negative in all samples from the patients with HSVE. The non-HSVE cases were initially suspected of having HSVE but were later proved to have other diseases. The diagnosis in non-HSVE patients was confirmed retrospectively to be fungal meningoencephalitis (two CSFs in two patients), acute disseminated encephalomyelitis (eight CSFs in six patients), rubella virus encephalitis (one CSF in one patient), Coxsackie encephalitis (four CSFs in two patients), herpes zoster virus encephalitis (two CSFs in two patients), and acute encephalitis of unknown aetiology (12 CSFs in seven patients). By the end of September 1998, the materials had been classified as $24 \mathrm{CSF}$ samples from 11 patients with serologically definite HSVE, and 29 CSF samples from 20 patients with acute non-HSVE. All the patients with HSVE due to HSV-1 were retrospectively confirmed by Southern blot hybridisation. After the patients had been classified according to the above mentioned serological criteria, the sensitivities, specificities, and predictive values of the chemiluminescence assay, low sensitive (single) PCR, and high sensitive (nested) PCR for the diagnosis of these serial cases of HSVE were evaluated. Comparisons of the values obtained for the three methods were made for significance by Fisher's exact probability test.

CHEMILUMINESCENCE ASSAY FOR QUANTITATIVE MEASUREMENT OF HSV ANTIGEN IN CSF

The chemiluminescence assay was carried out as described previously. ${ }^{1}$ Briefly, the chemiluminescence of normal granulocytes was measured in assays that included CSF and anti-HSV antibody. The assay mixture consisted of $0.1 \mathrm{ml}$ CSF sample, $0.7 \mathrm{ml}$ medium containing luminol, $0.1 \mathrm{ml}$ antihuman HSV antiserum, and 0.1

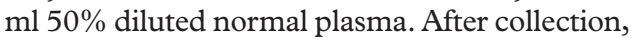
each CSF sample was stored at $-70^{\circ} \mathrm{C}$ for preservation of its viral antigenicity and inactivation of intrathecal granulocytes and macrophages. Normal plasma was isolated by centrifugation of heparinised normal human blood. This plasma was employed as complement in the present assay. The above mentioned mixture was incubated at $37^{\circ} \mathrm{C}$ for 30 minutes. After the incubation, $0.1 \mathrm{ml}$ normal granulocyte suspension was added. The chemiluminescence of the stimulated granulocytes was determined at $37^{\circ} \mathrm{C}$ with a luminescence reader (BLR-301; Aloka, Japan). The integrated value of the chemiluminescence for 4 minutes after supplementation of the normal granulocyte suspension was adopted. It took only 5 hours to complete this assay after obtaining the CSF sample. The increase in chemiluminescence values versus the
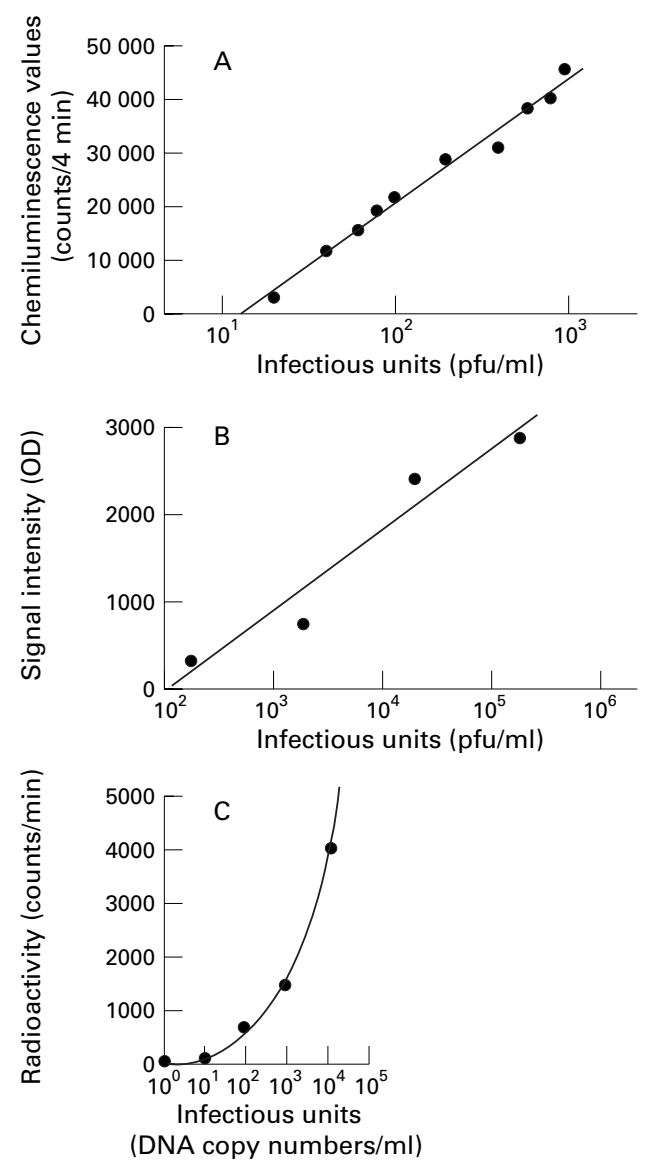

Figure 1 Quantitative virological studies by chemiluminescence assay, low sensitive PCR, and high sensitive PCR. The chemiluminescence values versus the common logarithm of the infectious units could be expressed in the form of a linear regression $(r=0.993)$. The low sensitive PCR yielded a linear regression versus the common logarithm of the known infectious units $(r=0.936)$. A quantitative study using the high sensitive PCR was undertaken based on the regression curve. The quantitative virological data for each of the three methods were obtained at different laboratories, independently. The data indicated only that the three procedures each have a potential as a quantitative method.

common logarithm of the infectious units (plaque forming units (pfu/ml) was expressed as a linear regression (figure $1 \mathrm{~A}$ ). The quantity of HSV antigen in the CSF was established from this regression line between the known infectious units and chemiluminescence values. The chemiluminescence value without addition of anti-HSV serum was negative. The error associated with the values in this assay was below $\pm 10 \%$, as described previously. ${ }^{1}$ The present assay represents a the method of substituting the quantity of chemiluminescence for the quantity of intrathecal viral antigen. This assay could detect not only HSV itself, but also the small fragment which was composed of viral component proteins with HSV antigenicity. ${ }^{1}$ If antigenantibody complexes were formed and complements were activated by the complexes in the above mentioned assay, chemiluminescence of reactive oxygen species from the stimulated granulocytes would be generated. Because the amount of anti-HSV antibody added was sufficient for the formation of antigen-antibody complexes in this chemiluminescence assay, the chemiluminescence values were regulated by the 
Results of quantitative studies employing chemiluminescence assay, low sensitive PCR, and high sensitive PCR in serial HSVE cases

\begin{tabular}{|c|c|c|c|c|c|}
\hline Patient No & $\begin{array}{l}\text { Days after onset of first } \\
\text { symptoms (days after onset of } \\
\text { neurological symptoms) }\end{array}$ & $\begin{array}{l}\text { Days after introduction of } \\
\text { acyclovir (Acv) and/or } \\
\text { adenine-arabinoside (ara-A) } \\
\text { treatment }\end{array}$ & $\begin{array}{l}\text { Quantity of HSV antigen by } \\
\text { chemiluminescence assay } \\
\text { (pfu/ml) }\end{array}$ & $\begin{array}{l}\text { Quantity of HSV selected } \\
\text { genome sequences by low } \\
\text { sensitive PCR (pfu/ml) }\end{array}$ & $\begin{array}{l}\text { Quantity of HSV } \\
\text { selected genome } \\
\text { sequences by high } \\
\text { sensitive PCR } \\
\text { (copies/ml) }\end{array}$ \\
\hline \multirow[t]{2}{*}{1} & $6(6)$ & $3(\mathrm{Acv})$ & 160 & - & not done \\
\hline & $14(14)$ & $11(\mathrm{Acv})$ and 8 (ara-A) & 120 & - & not done \\
\hline 2 & $16(13)$ & $13(\mathrm{Acv})$ & 50 & 50 & not done \\
\hline 3 & $10(10)$ & $7(\mathrm{Acv})$ & 160 & - & below 100 \\
\hline \multirow[t]{4}{*}{4} & $7(4)$ & Before treatment & 48000 & 47000 & 120000 \\
\hline & $15(10)$ & $9(\mathrm{Acv})$ & 4900 & 3500 & 9000 \\
\hline & $25(20)$ & $19(\mathrm{Acv})$ & 120 & - & 200 \\
\hline & $32(27)$ & $26(\mathrm{Acv})$ & - & - & - \\
\hline \multirow[t]{2}{*}{5} & $11(11)$ & $10(\mathrm{Acv})$ & 180 & - & below 100 \\
\hline & $18(18)$ & $17(\mathrm{Acv})$ & 120 & - & below 100 \\
\hline \multirow[t]{2}{*}{6} & $10(10)$ & $7(\mathrm{Acv})$ & 160 & - & - \\
\hline & $14(14)$ & $11(\mathrm{Acv})$ and 4 (ara-A) & 120 & - & - \\
\hline 7 & $10(10)$ & $9(\mathrm{Acv})$ & 50 & - & - \\
\hline 8 & $15(12)$ & $13(\mathrm{Acv})$ & 50 & - & - \\
\hline \multirow[t]{2}{*}{9} & $18(12)$ & $10(\mathrm{Acv})$ & 180 & - & - \\
\hline & $20(14)$ & $12(\mathrm{Acv})$ & 80 & - & - \\
\hline \multirow[t]{4}{*}{10} & $3(3)$ & Before treatment & 28000 & 21000 & 10000 \\
\hline & $5(5)$ & $5(\mathrm{Acv})$ & 32000 & 17000 & 20000 \\
\hline & $12(12)$ & $12(\mathrm{Acv})$ & 180 & - & - \\
\hline & $20(20)$ & $20(\mathrm{Acv})$ & - & - & - \\
\hline \multirow[t]{4}{*}{11} & $4(3)$ & Before treatment & 46000 & 30000 & 100000 \\
\hline & $11(10)$ & $11(\mathrm{Acv})$ & 6500 & - & 20000 \\
\hline & $22(21)$ & $22(\mathrm{Acv})$ & 200 & - & 200 \\
\hline & $32(31)$ & $32(\mathrm{Acv})$ & - & - & - \\
\hline
\end{tabular}

amount of HSV antigen, and not by the presence of anti-HSV antibody in CSF of the late stage and antibodies against common human viruses such as varicella zoster virus, mumps virus, and enterovirus - for example, Coxsackie virus. In fact, the chemiluminescence assay showed negative findings in the above mentioned other viral encephalitides with herpes zoster virus and Coxsackie virus, and in other forms of viral meningitis including that involving mumps and echo viruses, and control subjects (non-infectious neurological diseases). ${ }^{1}$

PCR FOR QUANTITATIVE DETECTION OF INTRATHECAL HSV SELECTED GENOME SEQUENCES

The low and high sensitive PCRs were carried out on the same CSF samples at different
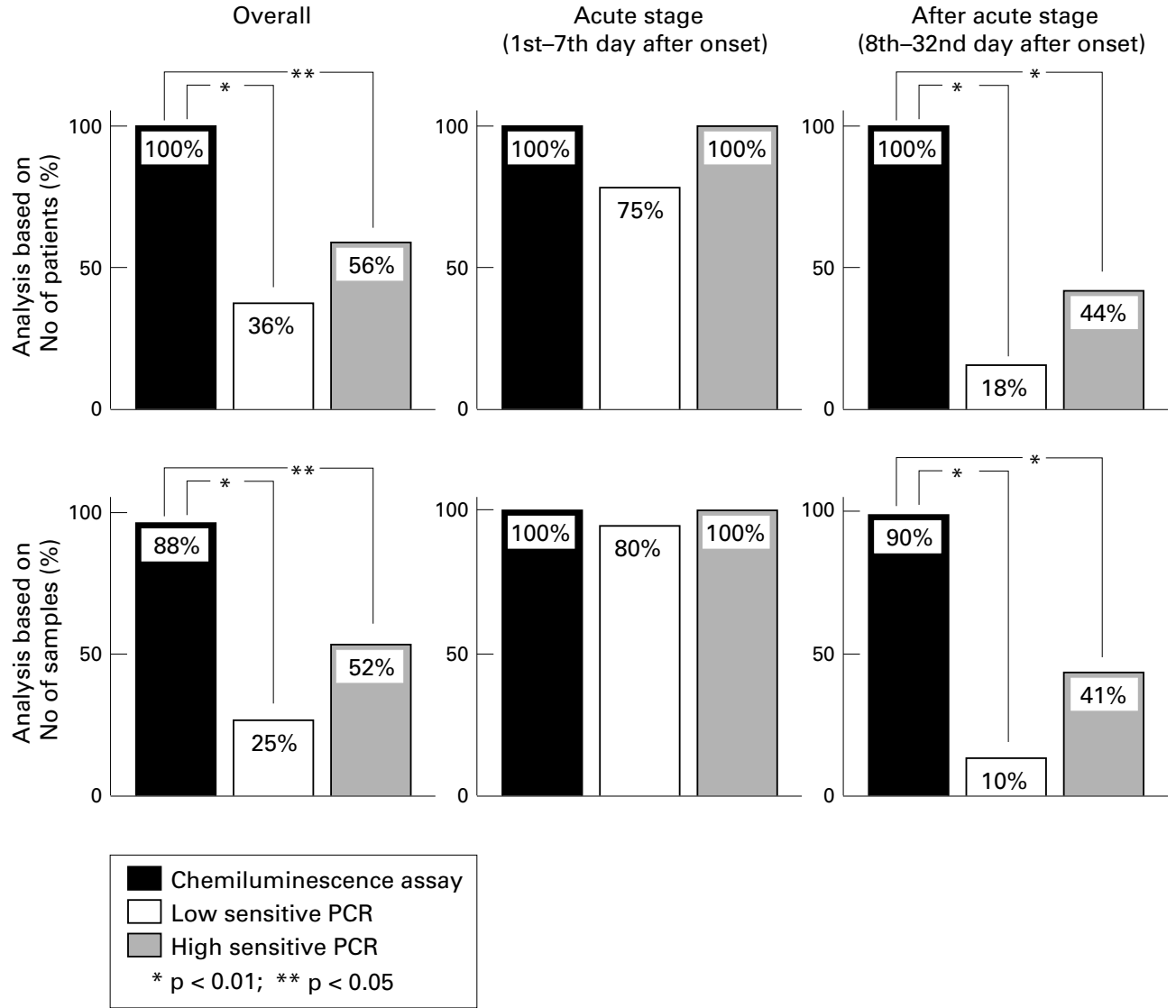

Figure 2 Comparative sensitivities of chemiluminescence assay, low sensitive PCR, and high sensitive PCR for the diagnosis of serial cases of HSVE. Probability was evaluated by Fisher's exact probability test. 

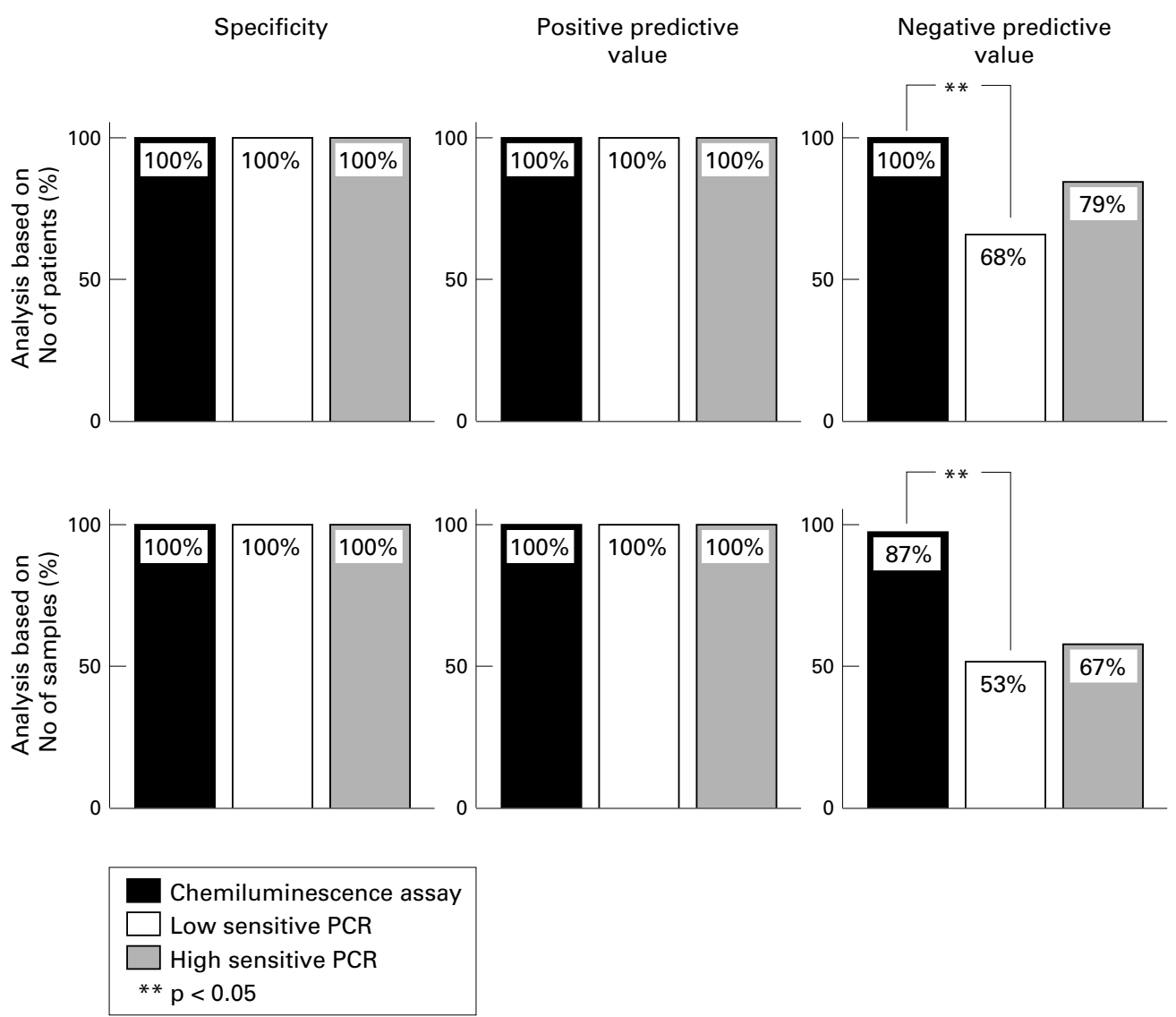

Figure 3 Specificities and positive and negative predictive values of chemiluminescence assay, low sensitive PCR, and high sensitive PCR. Probability was evaluated by Fisher's exact probability test.

laboratories. These PCRs could detect the intrathecal selected viral sequences of HSV-1 and 2 .

LOW SENSITIVE PCR

Each CSF sample stored at $-20^{\circ} \mathrm{C}$ was treated with lysis buffer containing proteinase $\mathrm{K}$. The DNA was extracted with phenol-chloroform and precipitated with ethanol. Oligonucleotide primers were chosen from the DNA polymerase gene. The upstream primer was 5'AGATGGCGAGCCACACATCTC-3' and the downstream primer was 5'CTCCGGATACGGTATCGTC-3'. The PCR was performed for 40 cycles, consisting of DNA denaturation at $94^{\circ} \mathrm{C}$ for 1 minute, primer annealing at $58^{\circ} \mathrm{C}$ for 2 minutes, and primer extension at $72^{\circ} \mathrm{C}$ for 2 minutes. The amplified DNA was subjected to electrophoresis on $1.5 \%$ agarose gel as a 234 bp sequence. The signal intensity of the PCR products was measured with a densitometer. The increase in intensity values versus the common logarithm of the infectious units was expressed as a linear regression (figure 1B). A quantitative study on the genome sequence was performed based on this regression line which was determined with samples including a known viral quantity. The minimum sensitivity of the above low sensitive PCR procedure was $50 \mathrm{pfu} / \mathrm{ml}$ in the CSF.

HIGH SENSITIVE PCR

The high sensitive (nested) PCR and Southern blot hybridisation were undertaken as de- scribed previously except that different primers were used. ${ }^{7}$ Briefly, each CSF sample stored at $-70^{\circ} \mathrm{C}$ was treated with lysis buffer containing proteinase $\mathrm{K}$. The DNA was extracted with phenol-chloroform and precipitated with ethanol. Two sets of primers were chosen from the DNA polymerase gene. The outer primers were 5'-CAGTACGGCCCCGAGTTCG TGACCGGG-3' (PR-1) and 5'-GGCGT AGTAGGCGGGGATGTCGCG-3' (PR-2). The inner primers were 5'-TACAAC ATCATCAACTTCGACTGG-3' (PR-3) and 5'-CCTTCTTCTTGTCCTTCAGG ACGG-3' (PR-4). The PCR was performed for 35 cycles, consisting of DNA denaturation at $96^{\circ} \mathrm{C}$ for 1 minute, primer annealing at $67^{\circ} \mathrm{C}$ for 2 minutes, and primer extension at $72^{\circ} \mathrm{C}$ for 3 minutes. The DNA was subjected to electrophoresis on 5\% agarose gel as a 330 bp sequence. The amplified products were transferred to a nylon membrane and hybridised with a ${ }^{32} \mathrm{P}$-labelled probe, 5'-AT GGTGAACATCGACATGTACGG-3'. The radioactivity of the PCR products on Southern blots was estimated with an image analysing system (Fujix BAS 2000; Fuji Photo Film Co, Japan). A quantitative study on the genome sequence was undertaken based on the regression curve (figure 1C). The minimum sensitivity of this high sensitive PCR procedure was 20 to 50 copies $/ \mathrm{ml}$ in the CSF.

The quantitative virological data for each of the three methods were obtained at different laboratories, independently. The PCR detects 
selected viral sequences, whereas the chemiluminescence assay detects viral fragment with antigenicity which was composed of viral protein. As there is a difference in principle between the PCR and chemiluminescence assay, analysis of the relations and direct comparisons between the quantitative values yielded by the different methods were impossible.

\section{Results}

The results of our quantitative studies employing the chemiluminescence assay, low sensitive PCR, and high sensitive PCR in the serial patients with HSVE are summarised in the table. The chemiluminescence assay showed 50 to $48000 \mathrm{pfu} / \mathrm{ml}$ in all of the CSF samples (except one) taken from the 3rd to 25th day of illness. The low sensitive PCR demonstrated 50 to $47000 \mathrm{pfu} / \mathrm{ml}$ in only six samples involving four out of the 11 patients with HSVE. The low sensitive PCR failed to detect any product in any of the other CSF samples. The high sensitive PCR disclosed less than 100 to 120000 copies $/ \mathrm{ml}$ in $11 \mathrm{CSF}$ samples involving five out of nine patients with HSVE. All CSF samples from non-HSVE patients yielded negative results by the three methods. The comparative sensitivity of the methods is illustrated in fig 2 . At the acute stage from the 1 st to 7 th day, the sensitivities of the chemiluminescence assay and high sensitive PCR were both 100\%. However, that of the low sensitive PCR was $75 \%$ even at the acute stage. The sensitivity of the chemiluminescence assay was significantly higher than those of both PCR methods after the acute stage on the 8th to the 32nd day. Different results between the low and high sensitive PCRs were obtained in six samples from four patients with HSVE. The specificities and positive predictive values of the three methods were $100 \%$ (fig 3). However, the negative predictive value of the chemiluminescence assay was significantly higher than that of the low sensitive PCR (figure 3).

\section{Discussion}

Comparisons between quantitative detection by the chemiluminescence assay and by the PCR for the same CSF samples of HSVE have remained problematical. ${ }^{12}$ Because the present prospective comparative study aimed to evaluate the relative methodological differences of the chemiluminescence assay and two different sensitive PCRs for the same CSF samples from serial HSVE cases, the diagnosis of HSVE was based on serological intrathecal antibody synthesis. The specificity of intrathecal antibody synthesis using the antibody ratio and C-EIA after the acute stage of HSVE has been reported to be $80 \%{ }^{9}$ and $95 \% .^{11}$ The rate of presence of pseudopositivity in evaluations of intrathecal antibody synthesis by both the antibody ratio and C-EIA methods was thus considered to be $1 \%$. Moreover, varicella zoster viral antibody, which confounds the results of EIA, ${ }^{11}$ was undetectable in all of the CSF specimens obtained from our cases of HSVE. The present retrospective clinical diagnosis of HSVE based on intrathecal antibody synthesis was thus regarded as highly reliable. The high sensitive PCR in our study showed sufficient potential to detect intrathecal viral selected sequences compared with a recent previously reported PCR. ${ }^{13}$

Our results for CSF samples at the acute stage of HSVE demonstrated the sensitivity of the chemiluminescence assay and that of the high sensitive PCR to be $100 \%$. Moreover, the quantitative values for the chemiluminescence assay correlated well with those for the high sensitive PCR. The sensitivity of the chemiluminescence assay is therefore considered to be equivalent to that of the high sensitive PCR at the acute stage of HSVE. On the other hand, the sensitivity of the chemiluminescence assay after the acute stage of HSVE was significantly higher than that of the high sensitive PCR. The reasons for this difference in results between the two methods in the case of CSF samples after the acute stage could be related to the disappearance from the CSFs of the selected viral genome sequence amplified with the high sensitive PCR. Disappearance of the intrathecal viral genome sequence using the PCR has been reported to occur from the 5 th day after onset of neurological symptoms in HSVE. ${ }^{414}$ The sensitivity of the low sensitive PCR had an unsatisfactorily low value even at the acute stage. A clear difference existed between the sensitivities of the two different PCR procedures in our serial cases of HSVE. It has also been reported previously by others that different results were obtained by different procedures of PCR with the same CSF samples in HSVE. ${ }^{15}$ The diagnostic potential of the PCR for HSVE is evidently influenced by the actual procedure of PCR, especially the minimum detection sensitivity of the PCR. One possible strategy is that the indication for anti-HSV treatment should be based on the results of the PCR for CSF samples. ${ }^{76}$ However, this could pose serious problems in the clinical diagnosis of HSVE, due to the risk of missing some patients with HSVE. Recommendations for performing the high sensitive (nested) PCR and for undertaking a combination of the PCR and serological diagnosis were thus given in a recent consensus regarding the management of suspected HSVE in the European Union. ${ }^{17}$ Under such conditions, however, standardisation of the PCR procedure for the diagnosis of HSVE would be required. Because PCR standardisation across many different laboratories is considered difficult to achieve, quantitative measurements of intrathecal genome sequences and a decision on the minimum sensitivity for the PCR in HSVE might be useful for making evaluations that cover the results of different PCR procedures.

On the other hand, our chemiluminescence assay was able to detect the intrathecal viral antigen in all CSF samples except one on the 3 rd to 25 th day. This finding suggests that the chemiluminescence assay has a high applicability to CSF samples not only during the acute stage but also after the acute stage. The chemiluminescence assay evidently possesses a high potential for early diagnosis, quantitative studies, and demonstrating a persistent positivity 
after the acute stage. However, this assay could detect only HSV-1, whereas the PCR could detect HSV-1 and HSV-2. In this regard, the PCR enjoys an advantage over the chemiluminescence assay. Thus in view of the merits and demerits of the different methods, the chemiluminescence assay has a strong potential as a supportive diagnostic method for the clinical diagnosis of HSVE. A combination of the PCR, chemiluminescence assay, and serological antibody diagnostic methods is currently considered the most effective approach for the clinical diagnosis of HSVE.

We are indebted to Professor E J Thompson, Department of Neuroimmunology, The National Hospital of Neurology and Neurosurgery, Queen Square, London, UK, for his invaluable comments regarding the manuscript. We are also indebted to D Y Sakai, Department of Internal Medicine, Surugadai Nihon University Hospital, Tokyo, Japan, Dr Y Tada, Department of Neurology, Eisei Hospital, Tokyo, Japan, and Dr T Yosizawa, Department of Internal Medicine, Kanamechou Hospital, Tokyo, Japan, for providing the CSF samples and the details of the patient data for registered HSVE patients. This study was supported by a grant in aid for scientific research (C) No 10670608 ) from the Ministry of Education, Science and Culture, Japan.

1 Kamei S, Tetsuka T, Takasu T, et al. New non-invasive rapid diagnosis of herpes simplex virus encephalitis by quantitative detection of intrathecal antigen with a chemiluminestive detection of intrathecal antigen with a chemilumines-
cence assay. F Neurol Neurosurg Psychiatry 1994;57:1112-4.

2 Puchhammer-Stöckl E, Popow-Kraupp T, Heinz FX, et al. Puchhammer-Stöckl E, Popow-Kraupp T, Heinz FX, et al.
Establishment of PCR for the early diagnosis of herpes Establishment of PCR for the early diagnosis
simplex encephalitis. F Med Virol 1990;32:77-82.

3 Rowley AH, Whitley RJ, Lakeman FD, et al. Rapid detection of herpes-simplex-virus DNA in cerebrospinal fluid of patients with herpes simplex encephalitis. Lancet 1990;335 $440-1$

4 Aurelius E, Johansson B, Sköldenberg B, et al. Rapid diagnosis of herpes simplex encephalitis by nested polymerase chain reaction assay of cerebrospinal fluid. Lancet 1991;337:189-92.

5 Lakeman FD, Whitley RJ, and the National Institute of Allergy and Infectious Diseases Collaborative Antiviral
Study Group. Diagnosis of herpes simplex encephalitis: application of polymerase chain reaction to cerebrospinal fluid from brain-biopsied patients and correlation with disease. F Infect Dis 1995;171:857-63.

6 Katz JP, Bodin ET, Coen DM. Quantitative polymerase chain reaction analysis of herpes simplex virus DNA in ganglia of mice infected with replication-incompetent mutants. F Virol 1990;64:4288-95.

7 Ando Y, Kimura H, Miwata H, et al. Quantitative analysis of herpes simplex virus DNA in cerebrospinal fluid of children with herpes simplex encephalitis. $f$ Med Virol 1993;41:170-3.

8 Wildmann B, Ehrhart K, Storch-Hagenlocher B, et al. Quantitation of herpes simplex virus type 1 DNA in cells of cerebrospinal fluid of patients with herpes simplex virus encephalitis. Neurology 1997;48:1341-6.

9 Kahlon J, Chatterjee S, Lakeman FD, et al. Detection of antibodies to herpes simplex virus in the cerebrospinal fluid of patients with herpes simplex encephalitis. F Infect Dis 1987; 155:38-44.

10 Klapper PE, Laing I, Longson M. Rapid non-invasive diagnosis of herpes encephalitis. Lancet 1981;ii: 607-9.

11 Aurelius E, Forsgren M, Skoog E, et al. Serodiagnosis of herpes simplex encephalitis by antibody capture enzymelinked immunosorbent assay. Serodiagnosis Immunotherapy In Infectious Disease 1989;3:249-58.

12 Snydman DR. Herpesviruses. Viral infections. In: Keusch GT, Barza MJ, eds. Year book of infectious diseases 1996. St Louis: Mosby Year Book, 1996:121-3.

13 Domingues RB, Lakeman FD, Mayo MS, et al. Application of competitive PCR to cerebrospinal fluid samples from patients with herpes simplex encephalitis. $\mathcal{F}$ Clin Microbiol 1998;36:2229-34.

14 Pohl-Koppe A, Dahm C, Elgas M, et al. The diagnostic significance of the polymerase chain reaction and isoelectric focusing in herpes simplex virus encephalitis. $7 \mathrm{Med}$ Virol 1992;36:147-54.

15 Klapper PE, Cleator GM, Tan SV, et al. Diagnosis of herpes simplex encephalitis with PCR. Lancet 1993;341:691.

16 Nakayama T, Sakaguchi T, Kikumoto O, et al. Rapid diagnosis and therapeutic evaluation of herpes simplex encephalitis using polymerase chain reaction from cerebrospinal fluids. Clin Neurol 1994;34:170-3. (In Japanese.)

17 Cinque $\mathrm{P}$, Cleator GM, Weber T, et al, for the EU concerted action on virus meningitis and encephalitis. The role of laboratory investigation in the diagnosis and management of patients with suspected herpes simplex encephalitis: a consensus report. $\mathcal{F}$ Neurol Neurosurg Psychiatry 1996;61: $339-45$. 